\title{
Experimental Investigation and Passive Flow Control of a Cavitating Centrifugal Pump
}

\author{
Spyridon D. Kyparissis and Dionissios P. Margaris \\ Fluid Mechanics Laboratory, Mechanical Engineering and Aeronautics Department, University of Patras, 26500 Patras, Greece
}

Correspondence should be addressed to Spyridon D. Kyparissis, kypariss@mech.upatras.gr

Received 17 July 2012; Revised 15 November 2012; Accepted 6 December 2012

Academic Editor: N. Sitaram

Copyright ( $) 2012$ S. D. Kyparissis and D. P. Margaris. This is an open access article distributed under the Creative Commons Attribution License, which permits unrestricted use, distribution, and reproduction in any medium, provided the original work is properly cited.

\begin{abstract}
Passive flow control techniques are used to improve the flow field and efficiency of centrifugal pumps and turbomachines, in general. An important phenomenon that mechanical engineers have to take into account is cavitation. It leads to the decrease of the pump performance and total head. In the present experimental study, a centrifugal pump is investigated in cavitating conditions. A passive flow control is realized using three different blade leading edge angles in order to reduce the cavitation development and enhance the pump performance. The experiments are carried out in a pump test rig specially designed and constructed, along with the impellers. The head drop and total efficiency curves are presented in order to examine the effect of the blade leading edge angle on the cavitation and pump performance. Finally, the vapour distribution along with the blades is illustrated for the tested blade leading edge angles.
\end{abstract}

\section{Introduction}

Cavitation is a very complex phenomenon including phase changes and viscous effects. When the local pressure within a flow with water as medium becomes equal to the vaporization pressure of the water, bubbles begin to form and are further transported to regions of higher pressure where they collapse. This bubble collapse can cause material damage and erosion.

Various researchers have considerably contributed in studying the cavitation in centrifugal pumps, applying passive flow control techniques and realizing a series of experiments. On the other hand, there is a limited number of works regarding the effect of the blade leading edge angle on the pump cavitation. Hirschi et al. [1] presented the results obtained with a $3 \mathrm{D}$ numerical method allowing the prediction of the cavitation behaviour of a centrifugal pump and compared this prediction to model tests. Hofmann et al. [2] studied experimentally the cavitation of two centrifugal pumps that can hold different runner geometries as well as different leading edge geometries within the same runner. Frobenius et al. [3] realized numerical simulations and experimental investigations of the cavitating flow through a centrifugal pump impeller of low specific speed. CoutierDelgosha et al. [4] investigated a special test pump with two-dimensional curvature blade geometry in cavitating and noncavitating conditions using different experimental techniques.

Siljegovic et al. [5] concluded that passive flow control can effectively modulate flow conditions in a capillary driven microfluidic device. Ulas [6] designed and studied experimentally two cavitating venturis to deliver the desired mass flow rates for specific conditions. Escaler et al. [7] carried out an experimental investigation in order to evaluate the detection of cavitation in actual hydraulic turbines. Japikse et al. [8] realized a series of tests and improved the stability of compressors and turbopumps using passive flow control. Luo et al. [9] studied experimentally the effect of impeller inlet geometry on performance improvement for a centrifugal pump. Wu et al. [10] carried out an experimental study in order to analyze the cavitation of a centrifugal pump and its effect on hydrodynamic performance during transient operation. Kyparissis and Margaris [11-13] studied experimentally and computationally a centrifugal pump with double-arc synthetic blade design method in cavitating and noncavitating conditions, applying different rotational speeds. 
The present experimental study and passive flow control are concerned with the effect of the blade leading edge angle on the cavitation of a centrifugal pump applying the doublearc synthetic blade design method [14-16], with rotational speed of $1200 \mathrm{rpm}$ and flow rate of $35 \mathrm{~m}^{3} / \mathrm{h}$. The experiments are carried out in a pump test rig specially designed and constructed, along with the impellers. The test section allows optical observation of the flow field and cavitation with the aid of the stroboscopic light source. The head drop and total efficiency curves are presented in order to investigate the effect of the blade leading edge angle on the cavitation and pump performance. In addition, the vapour distribution along with the blades is depicted for the tested blade leading edge angles.

\section{Experimental Rig and Procedure}

The experimental study is performed using the pump test rig shown in Figure 1. The supplying tank (1) of the pumping installation is filled with water from the water supply. The pressure at the examined pump inlet is measured by the absolute pressure transducer (2). Furthermore, the study of cavitation is realized applying a wide range of pressures at the pump inlet that can be set by changing the pressure in the supply tank with the aid of a vacuum pump (3). The fluid temperature is measured by a temperature sensor (4) and is kept almost constant and equal to $25^{\circ} \mathrm{C}$. The fluid level in the supply tank is read from the level meter (5) and the fluid-free surface pressure is measured by a vacuum-pressure gauge (6). The pump flow rate is measured using the electromagnetic flowmeter (7). Moreover, the flow rate regulation is obtained by throttling a butterfly valve (8) installed at the discharge pipe. The differential pressure between the pump inlet and outlet is measured by the differential pressure transducer (9). The pump is driven by a three-phase AC electric motor (10) of $2.2 \mathrm{~kW}$ and maximum rotational speed of $1410 \mathrm{rpm}$. The rotational speed can be continuously varied by an inverter (11). The pump suction cover (12) and a part of the suction pipe (13) are made from plexiglas. Transparent parts of the pump test rig allow observation of fluid flow inside the impeller and photography of the development of cavitation in flow passage of the impeller. The measurement data are acquired by the data acquisition system and LabVIEW platform (14). The main dimensions of the pump test rig and the regions where the pressure transducers are installed are shown in Figure 2.

For the realization of the cavitation test, the vacuum pump is set in operation and the air is removed from the tank, until a specific value of pressure is recorded by the vacuum-pressure gauge for every measurement. The pump rotational speed is kept constant and the butterfly valve is kept in a given position in order to have constant flow rate. For every measurement, the total head is computed according to the following formula:

$$
H=\frac{\Delta p}{\rho g}+\Delta z+\frac{u_{d}^{2}-u_{s}^{2}}{2 g} .
$$

The differential pressure $\Delta p$ is measured by the differential pressure transducer (9). Moreover, the water density

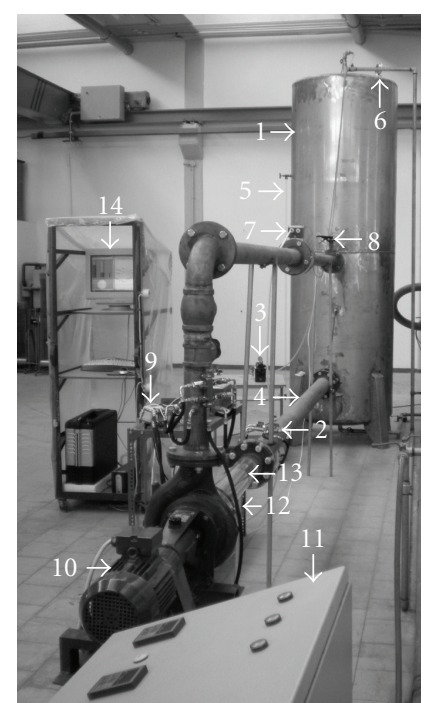

FIgURE 1: The complete experimental setup along with the instrumentation system.

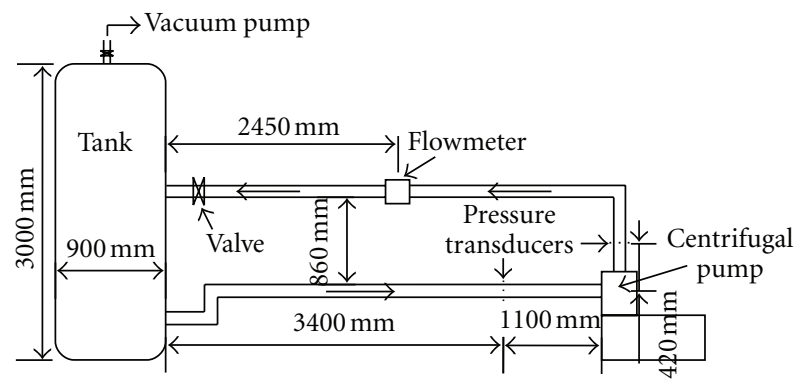

FIgURE 2: The main dimensions of the pump test rig.

for the temperature of $25^{\circ} \mathrm{C}$ is found $\rho=997 \mathrm{~kg} / \mathrm{m}^{3}$. The discharge velocity $u_{d}$ and the suction velocity $u_{s}$ are calculated according to the corresponding flow rate $Q$ measured by the electromagnetic flowmeter (7). The gravitational acceleration is determined as $g=9.807 \mathrm{~m} / \mathrm{s}^{2}$ and the elevation difference between the pump inlet and the region where the discharge pressure is measured is calculated $\Delta z=0.42 \mathrm{~m}$.

In addition, the net positive suction head available is computed according to the following equation:

$$
\mathrm{NPSHa}=\frac{p_{s}}{\rho g}+\frac{u_{s}^{2}}{2 g}-\frac{p_{v}}{\rho g} .
$$

The suction pressure $p_{s}$ is measured by the absolute pressure transducer (2) and the vaporization pressure of the water for the temperature of $25^{\circ} \mathrm{C}$ is found $p_{v}=3290.5 \mathrm{~Pa}$.

Finally, the total efficiency $\eta$ is computed according to the following expression:

$$
\eta=\frac{\rho g Q H}{\sqrt{3} V I} .
$$

The voltage $V$ and the amperage $I$ are measured by the inverter (11). 


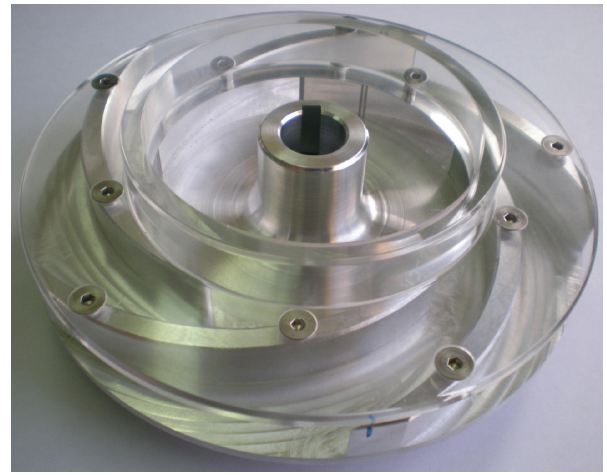

(a)

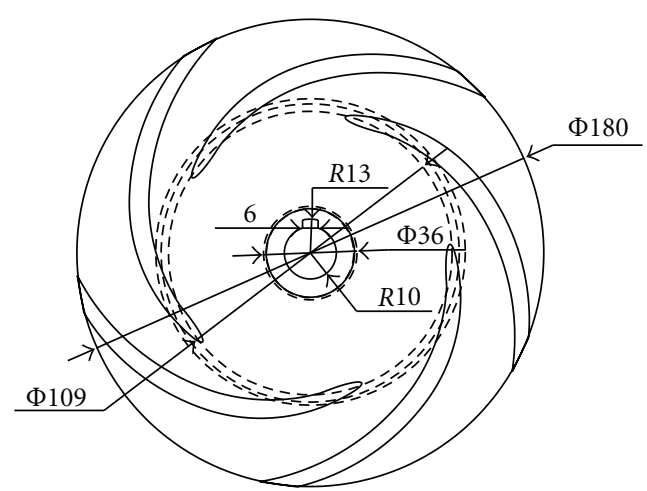

(b)

FIgURE 3: The examined impeller geometry and the scaled drawing (in $\mathrm{mm}$ ) with blade leading edge angle of 9 deg.

Moreover, during every measurement, the cavitation is illustrated using the stroboscopic light. The NPSH value that the first bubble appears, according to the visualization of cavitation, is the net positive suction head required NPSHr.

The measuring equipment consists of an absolute and a differential pressure transducer, a temperature sensor, and an electromagnetic flowmeter. In order to measure the static pressure at the suction side of the pump, an absolute pressure transducer from ABB 2600T series, model 264NS with a base accuracy $\pm 0.075 \%$ is applied. Moreover, the pressure difference between the discharge and suction region of the pump is measured using a differential pressure transducer from ABB $2600 \mathrm{~T}$ series, model 264DS with a base accuracy $\pm 0.075 \%$. In addition, temperature is measured with the temperature sensor of the ABB SensyTemp TSP111 series. Furthermore, the measurement of the flow rate is realized using an electromagnetic flowmeter ABB FXE4000 COPA$\mathrm{XE} / \mathrm{MAG}-\mathrm{XE}$, model DE $41 \mathrm{~F}$ with a base accuracy $\pm 0.5 \%$.

The rotational speed of the three phase electric motor can be continuously varied by the inverter Fuji Electric FVR-E9S series.

The study of cavitation is realized using the vacuum pump series LABOPORT N 816.3 KN.45.18. Series N 816.3 diaphragm pumps are double-head, dry-running devices used in a wide range of laboratory applications. They transfer and pump down without contamination.

The measurement data from the pressure transducers, flowmeter, and temperature sensor are acquired by the analog input module cFP-AI-110. The National Instruments cFP-AI-110 is an 8-channel single-ended input module for direct measurement of millivolt, low voltage, or milliampere current signals from a variety of sensors and transmitters. It delivers filtered low-noise analog inputs with 16-bit resolution and $5 \mathrm{~S} / \mathrm{s}$ sampling rate. Finally, this analog input module is connected to LabVIEW platform (14).

\section{Blade Design Analysis}

3.1. Impeller Geometry. Three impeller geometries have been constructed using aluminium alloy 7075-T6, which is composed of zinc as the primary alloying element. It is strong, with strength comparable to many steels and has
TABle 1: Main characteristics of the tested impellers.

\begin{tabular}{lc}
\hline Impeller characteristics & Values \\
\hline Suction pipe diameter $D_{s}, \mathrm{~mm}$ & 100 \\
Diameter of the impeller at the suction side $D_{1}, \mathrm{~mm}$ & 108 \\
Diameter of the impeller at the pressure side $D_{2}, \mathrm{~mm}$ & 180 \\
Impeller width at the suction side $b_{1}, \mathrm{~mm}$ & 15.6 \\
Impeller width at the pressure side $b_{2}, \mathrm{~mm}$ & 15.6 \\
Blade leading edge angle $\beta_{1}$, deg & $9,15,21$ \\
Blade trailing edge angle $\beta_{2}$, deg & 20 \\
Maximum blade thickness $S, \mathrm{~mm}$ & 7.5 \\
Number of blades $z$ & 5 \\
\hline
\end{tabular}

good fatigue strength and average machinability, but has less resistance to corrosion than many other aluminium alloys. 7075-T6 is a heat temper grade of aluminium alloy 7075. It has an ultimate tensile strength of 510-538 MPa and yield strength of at least $434-476 \mathrm{MPa}$. In addition, the tested impellers have a removable transparent cover disk made of plexiglas in order to observe the fluid flow and the cavitation, as shown in Figures 3, 4, and 5.

The impellers have been designed according to the dimensions of the Caprari MEC-A2/80A volute, which is installed in the pump test rig. The main characteristics of the three tested impellers are presented in Table 1.

3.2. Double-Arc Synthetic Method: DASM. In the present work, the double-arc synthetic method (DASM) [14-16] is applied for the impellers blade design of the centrifugal pump. It is a simple design method that combines two Pfleiderer's double-arc methods [17]. There are published computational studies [14-16] that compare DASM with the Pfleiderer's [17] methods and it is observed that a centrifugal pump has better efficiency applying DASM.

As shown in Figure 6, the auxiliary circle $C_{a}$ is drawn, according to the following formula:

$$
d_{1}=D_{1} \sin \beta_{1},
$$

where $D_{1}$ is the diameter of the impeller at the suction side and $\beta_{1}$ is the blade leading edge angle. 


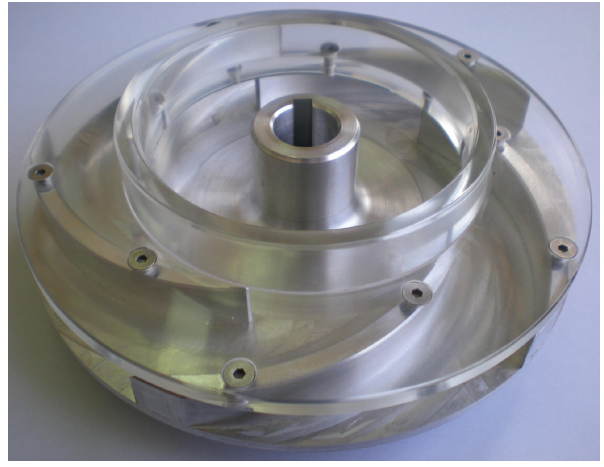

(a)

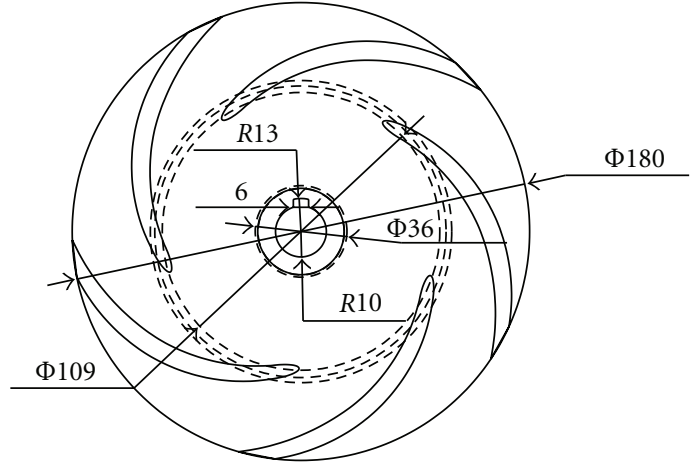

(b)

Figure 4: The examined impeller geometry and the scaled drawing (in mm) with blade leading edge angle of 15 deg.

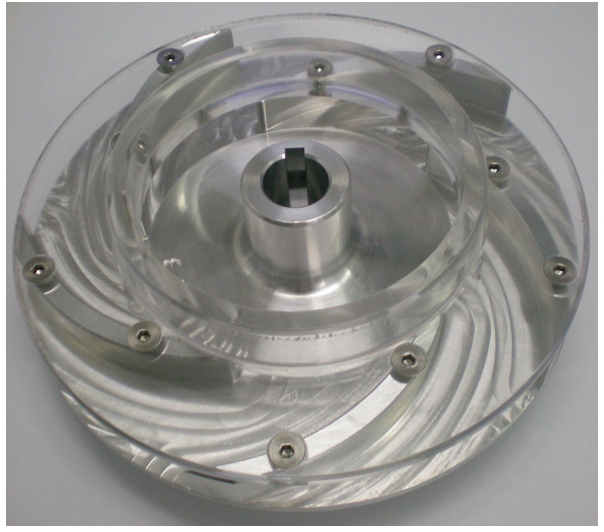

(a)

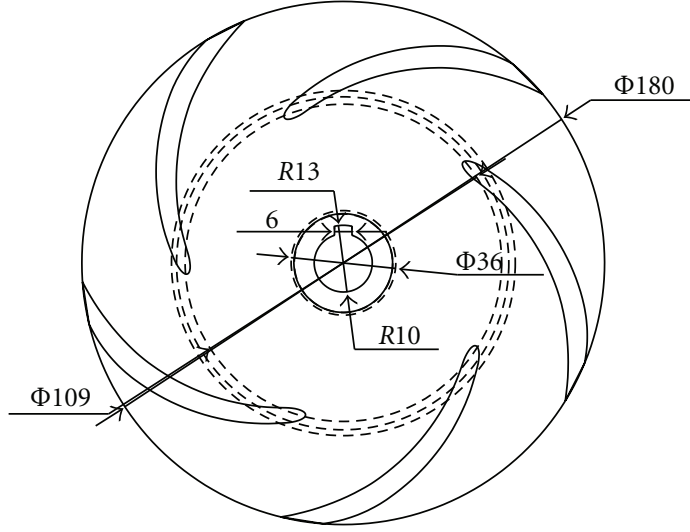

(b)

Figure 5: The examined impeller geometry and the scaled drawing (in $\mathrm{mm}$ ) with blade leading edge angle of $21 \mathrm{deg}$.

The periphery of the suction side is divided to five parts, just as the number of blades $z$. The tangents of the auxiliary circle from the points $\mathrm{E}$ and $\mathrm{C}$ are intersected to the point A. Afterwards, point $\mathrm{A}$ is shifted to the point $\mathrm{L}$, which is the center of curvature for the first arc EY and the radius $R_{1}$ is the distance of the points $\mathrm{E}$ and $\mathrm{L}$, as formulated in the following expression:

$$
R_{1}=1.2(\mathrm{AE}) .
$$

The point $\mathrm{P}$ is at the extension of the line that connects the points $\mathrm{A}$ and $\mathrm{C}$ at distance equal to the distance of the points A and E. Applying DASM the point $\mathrm{P}$ is shifted to $\mathrm{Y}$, according to the following expression:

$$
\mathrm{EY}=0.75 \mathrm{EP} .
$$

The angle between the lines OY and LY is defined as $\beta$ and the distance between the points $\mathrm{O}$ and $\mathrm{Y}$ is defined as $r$, as shown in Figure 6. The radius $R_{2}$ of the second arc $\mathrm{YH}$ is the distance of the points $\mathrm{K}$ and $\mathrm{Y}$ and is obtained from the following expression:

$$
R_{2}=\frac{1}{2} \frac{\left(D_{2} / 2\right)^{2}-r^{2}}{\left(D_{2} / 2\right) \cos \beta_{2}-r \cos \beta},
$$

where $D_{2}$ is the diameter of the impeller at the pressure side and $\beta_{2}$ is the blade trailing edge angle.

\section{Experimental Results}

In the present study, the experimental investigation and passive flow control are realized in order to examine the pump performance and cavitation development for three different blade leading edge angles. Studying the noncavitating performance, the total head and the total efficiency as functions of the flow rate are illustrated in Figures 7 and 8, for the three examined blade leading edge angles and rotational speed of $1200 \mathrm{rpm}$. As shown in Figure 8, the flow rate to the best efficiency point is approximately $22 \mathrm{~m}^{3} / \mathrm{h}$ for $9 \mathrm{deg}$, $34 \mathrm{~m}^{3} / \mathrm{h}$ for $15 \mathrm{deg}$, and $43 \mathrm{~m}^{3} / \mathrm{h}$ for $21 \mathrm{deg}$.

The total head drops for the rotational speed of $1200 \mathrm{rpm}$, flow rate of $35 \mathrm{~m}^{3} / \mathrm{h}$, and three examined blade leading edge angles of 9,15 , and $21 \mathrm{deg}$ are depicted in Figure 9. The ordinate is the total head of the pump, while the abscissa is the net positive suction head available. There are four capital letters in parentheses corresponding to the vapour distributions in Figures 12, 13, 14, and 15. The filled points represent the experimental cases, where cavitation has been developed. It is noticed that as the NPSHa decreases the total head decreases slowly for all the tested blade leading edge angles. As the water in the suction pipe approaches the impeller eye, it has velocity and acceleration. In addition, it has to change its direction to enter the impeller, because 


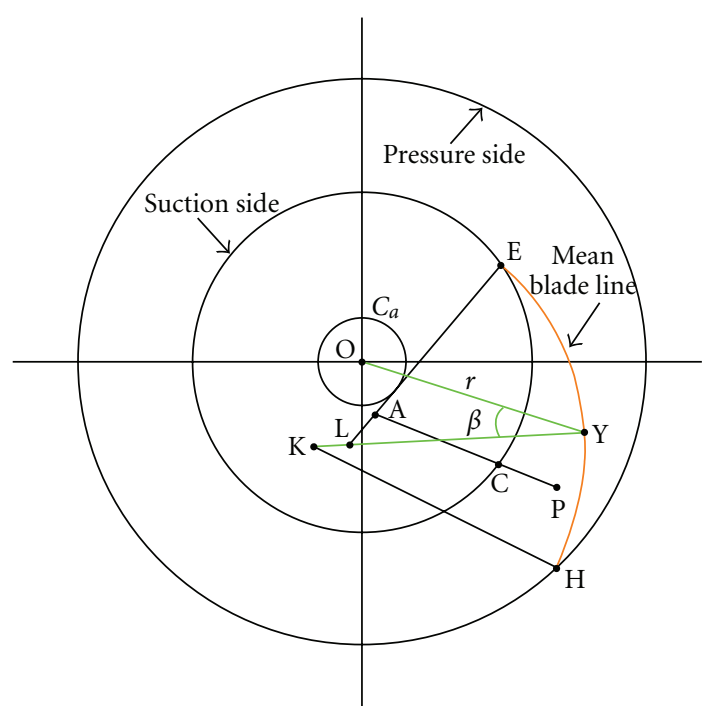

Figure 6: Representation of the mean blade line using DASM.

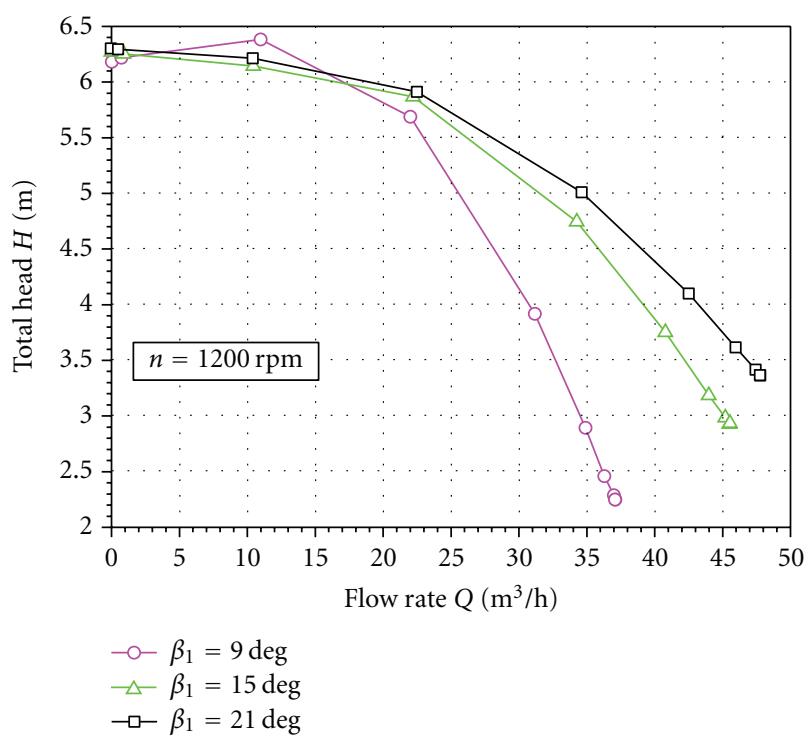

Figure 7: The total head as a function of the flow rate, studying the noncavitating performance.

of the blade leading edge angle. Losses in terms of total head occur due to each of the above reasons and because of friction. For the blade leading edge angle of $15 \mathrm{deg}$, no cavitation is noticed, for the examined pressures at the pump inlet. Nevertheless, there is head drop as the NPSHa decreases, because there are cavitation bubbles, very small and cannot be observed using the stroboscopic light. These bubbles block the flow passage between the blades and cause the head drop. Examining the blade leading edge angle of 9 deg, cavitation starts to be developed for the net positive suction head of $5.13 \mathrm{~m}$, which is the corresponding NPSHr for $9 \mathrm{deg}$. In addition, testing the $21 \mathrm{deg}$, the cavitation begins for $\mathrm{NPSH}=2.29 \mathrm{~m}$, which is the corresponding NPSHr for $21 \mathrm{deg}$. Realizing a series of experiments the maximum total head drops for the three above mentioned studied

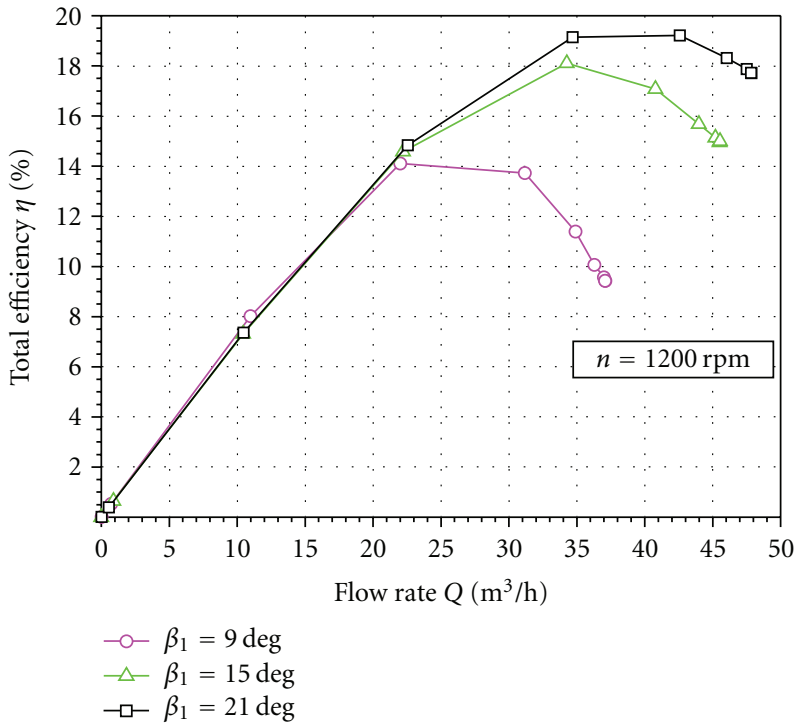

FIGURE 8: The total efficiency as a function of the flow rate, studying the noncavitating performance.

blade leading edge angles are $13.32 \%, 4.46 \%$, and $6.80 \%$, respectively. For the blade leading edge angles 9 and $21 \mathrm{deg}$, when cavitation starts to be developed the corresponding head drops are $5.47 \%$ and $5.49 \%$, respectively. Thus, it is observed that for both the cases that cavitation begins, the total head is reduced by $5.5 \%$ instead of $3 \%$ that the Hydraulic Institute defines. The NPSHr can be from 2 to 20 times the 3\% that the Hydraulic Institute defines, depending on pump design [18]. The cavity length when the cavitation bubbles appear is measured 4 and $7 \mathrm{~mm}$ for 9 and $21 \mathrm{deg}$, respectively. Moreover, it is noticed that as the blade leading edge angle increases the total head increases as well. Finally, as the net positive suction head available decreases the pump flow rate is kept almost constant, in spite of the cavitation development.

Furthermore, in Figure 10 the change of the total head with the blade leading edge angle is depicted, for three different values of the net positive suction head available. Figure 10 contributes to the recording of the total head values in respect of the change of the blade leading edge angle. It is observed that as the blade leading edge angle increases, the total head increases as well. For the blade leading edge angles between 15 and $21 \mathrm{deg}$, the total head is almost the same for the net positive suction head available values of 6 and $9 \mathrm{~m}$. Finally, for the lowest examined value of the net positive suction head available, the total head has the lowest values for all the examined blade leading edge angles, because as the net positive suction head decreases the total head decreases as well, as shown in Figure 9.

In Figure 11 the change of the total efficiency with the net positive suction head available is presented, for the three examined blade leading edge angles, rotational speed of $1200 \mathrm{rpm}$, and flow rate of $35 \mathrm{~m}^{3} / \mathrm{h}$. It is observed that the change of the total efficiency with the NPSHa is similar to the change of the total head with the NPSHa, as shown in 


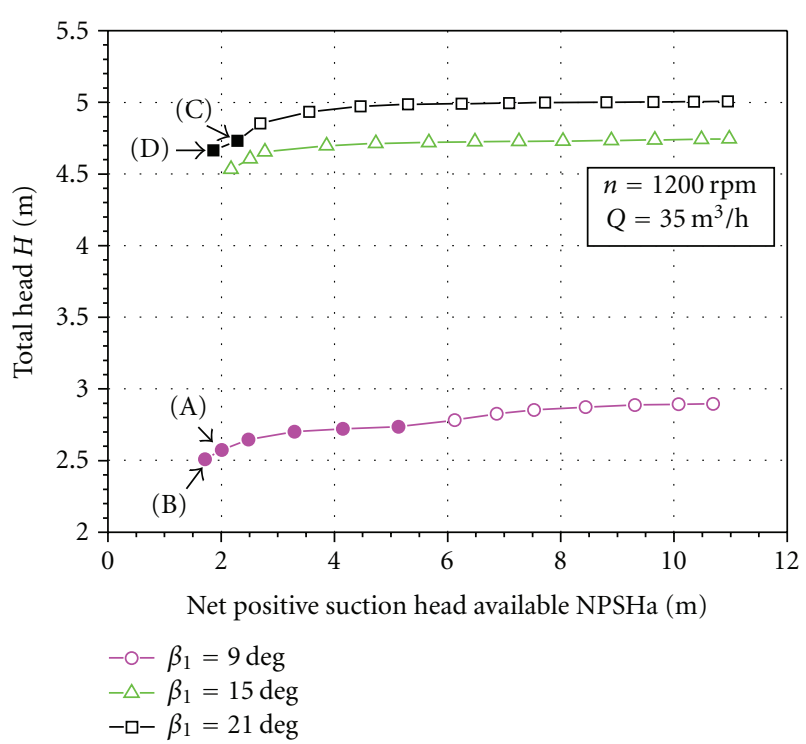

Figure 9: Total head drop curves, where cavitation has been developed to the filled points.

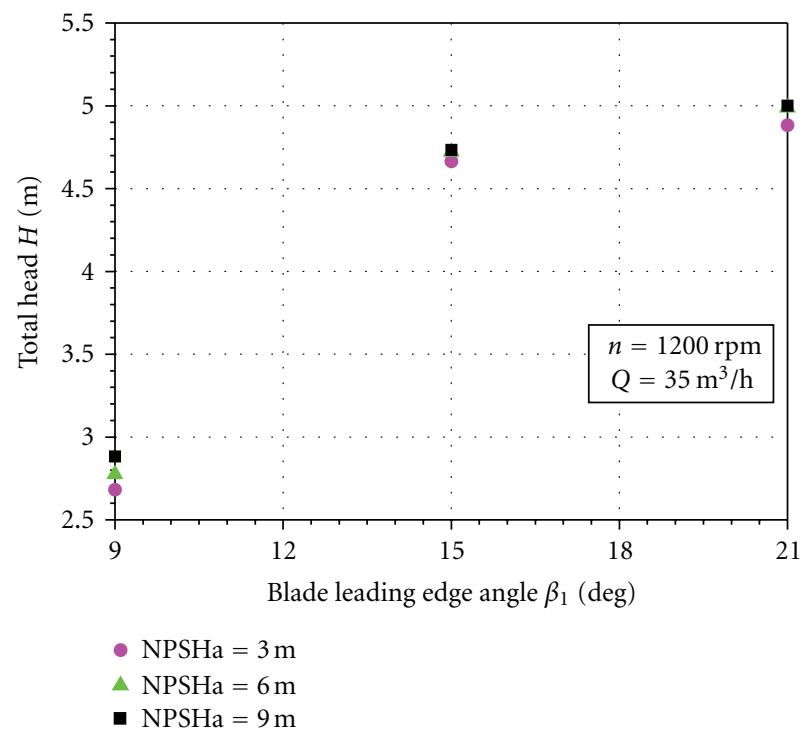

Figure 10: The change of the total head with the blade leading edge angle for three different values of the net positive suction head available.

Figure 9. As the blade leading edge angle increases the total efficiency increases as well. In addition, for the lowest values of the NPSHa, much faster decrease of the total efficiency is observed, for all the tested blade leading edge angles. Examining the blade leading edge angles 15 and $21 \mathrm{deg}$, fluctuations are observed to the total efficiency, because of the unstable measurements of both the voltage and the amperage.

The uncertainty on the measurements has been evaluated from the resolution of the data acquisition system, realizing multiple experiments with deviation from the presented data, approximately $\pm 0.10 \%$. The relative uncertainty for the total head is $0.07 \%$ for all the examined blade leading

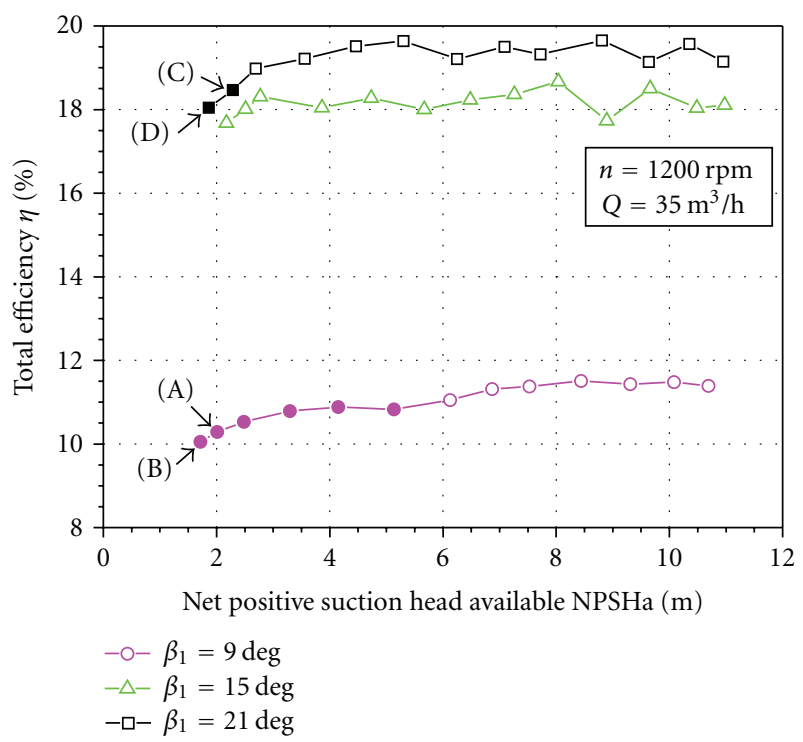

FIGURE 11: The change of the total efficiency with the net positive suction head available for rotational speed of $1200 \mathrm{rpm}$ and flow rate of $35 \mathrm{~m}^{3} / \mathrm{h}$.

edge angles. For the net positive suction head available, the relative uncertainty varies from $0.08 \%$ to $0.10 \%$ for $9 \mathrm{deg}$ and $21 \mathrm{deg}$ and $0.08 \%$ to $0.09 \%$ for $15 \mathrm{deg}$. Finally, for the total efficiency, the relative uncertainty varies from $0.54 \%$ to $0.55 \%$ for $9 \mathrm{deg}$ and is $0.53 \%$ for $15 \mathrm{deg}$ and $21 \mathrm{deg}$.

Furthermore, in the present experimental study the vapour distribution between the blades at the leading edge is illustrated using the stroboscopic light. The vapour is caused by the strong curvature of the streamlines along the front shroud, where low values of the net positive suction head prevail. Two of the cavitation cases of $9 \mathrm{deg}$ are depicted in Figures 12 and 13, where a scaling bar is added to each figure, representing a length of $10 \mathrm{~mm}$ and a letter is added in parentheses corresponding to the letter in Figures 9 and 11. Thus Figure 12 corresponds to the point A and Figure 13 corresponds to the point B. Testing the blade leading edge angle of $9 \mathrm{deg}$, the cavitation is observed at the leading edge in the region of the pressure side. Moreover, it is noticed that as the net positive suction head decreases, lower pressures between the blades prevail and the cavity length increases. As the cavity length increases the total head and total efficiency decrease, as shown in Figures 9 and 11, due to flow blockage from cavitation vapour in the blades.

In Figures 14 and 15 the cavitation is developed at the leading edge in the region of the suction side for the blade leading edge angle of $21 \mathrm{deg}$. Figure 14 corresponds to the point $\mathrm{C}$ and Figure 15 corresponds to the point $\mathrm{D}$. The region where the cavitation is developed is different from the corresponding of $9 \mathrm{deg}$. As the blade leading edge angle decreases, the passage between the blades decreases, lower pressures prevail in this region and the cavitation is developed at the pressure side. On the other hand, for blade leading edge angle of $21 \mathrm{deg}$, the lowest pressures prevail at the suction side of the blades and cavitation is developed in this region. Examining the blade leading edge angle of 


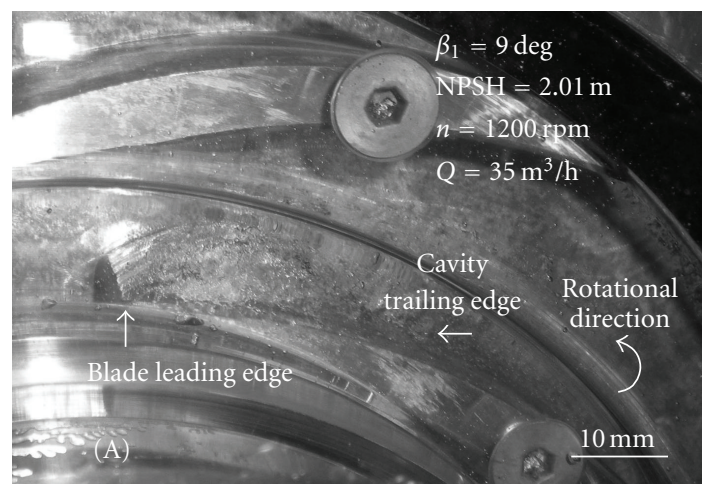

FIGURE 12: Illustration of cavitation for the blade leading edge angle of $9 \mathrm{deg}$ and NPSH equal to $2.01 \mathrm{~m}$.

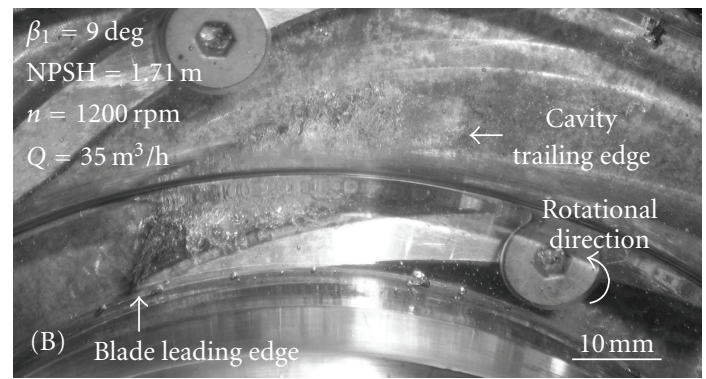

FIGURE 13: Illustration of cavitation for the blade leading edge angle of 9 deg and NPSH equal to $1.71 \mathrm{~m}$.

$15 \mathrm{deg}$, no cavitation is observed, because the testing flow rate of $35 \mathrm{~m}^{3} / \mathrm{h}$ is almost equal to the corresponding of the best efficiency point for $15 \mathrm{deg}$. For $9 \mathrm{deg}$, the testing flow rate of $35 \mathrm{~m}^{3} / \mathrm{h}$ is greater than its corresponding best efficiency point, thus the cavitation is developed at the pressure side of the blades. On the other hand, for $21 \mathrm{deg}$, the testing flow rate $35 \mathrm{~m}^{3} / \mathrm{h}$ is less than its corresponding best efficiency point and the cavitation is developed at the suction side of the blades. Moreover, the cavity length for $21 \mathrm{deg}$ is smaller than the corresponding of $9 \mathrm{deg}$, because lower net positive suction head values prevail for blade leading edge angle of 9 deg, as shown in Figures 9 and 11.

\section{Conclusions}

The present experimental study and passive flow control are concerned with the effect of the blade leading edge angle on the cavitation and performance of a centrifugal pump. A pump test rig has been developed in order to realize a passive flow control with different blade leading edge angles, allowing optical observation of the cavitation development with the aid of a stroboscopic light source.

The rotational speed of $1200 \mathrm{rpm}$ and flow rate of $35 \mathrm{~m}^{3} / \mathrm{h}$ are applied. Studying the effect of the blade leading edge angle on the cavitation, it is observed that as the blade leading edge angle increases, both the total head and the total efficiency increase for the examined operating conditions. Moreover, it is noticed that as the net positive suction head decreases the total head decreases slowly for all the examined blade leading edge angles.

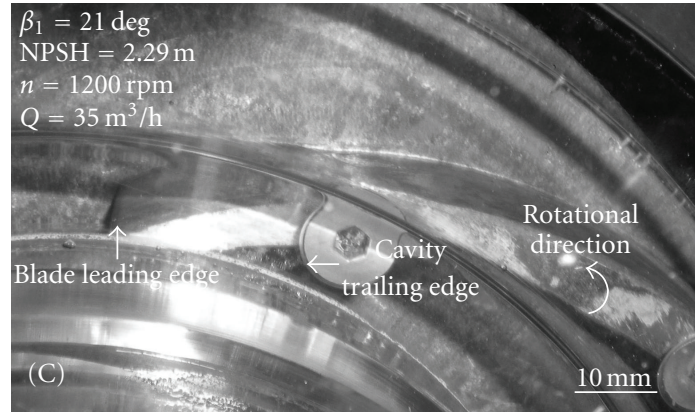

FIGURE 14: Illustration of cavitation for the blade leading edge angle of $21 \mathrm{deg}$ and NPSH equal to $2.29 \mathrm{~m}$.

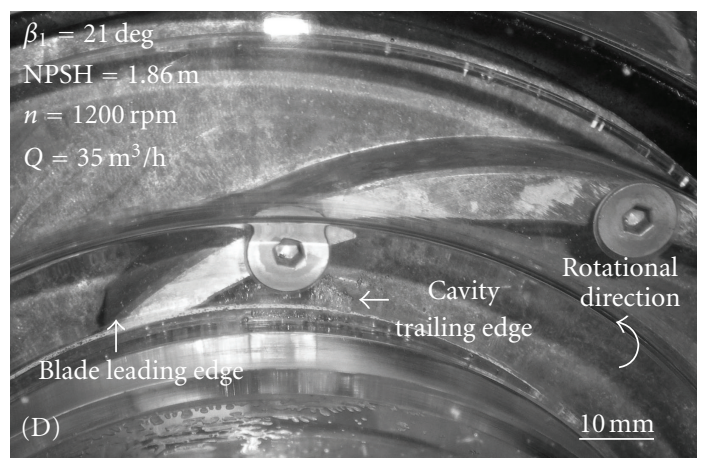

FIGURE 15: Illustration of cavitation for the blade leading edge angle of $21 \mathrm{deg}$ and NPSH equal to $1.86 \mathrm{~m}$.

Examining the blade leading edge angle of $9 \mathrm{deg}$, the cavitation is developed at the leading edge in the region of the pressure side. On the other hand, for $21 \mathrm{deg}$, the cavitation is developed at the leading edge in the region of the suction side. Finally, for the blade leading edge angle of $15 \mathrm{deg}$, there is no cavitation development and the danger of the pump damage and erosion is eliminated. Thus, the costs of maintenance of the centrifugal pumps are limited, applying the blade leading edge angle of $15 \mathrm{deg}$.

Further research work is planned to be accomplished experimentally and computationally, studying the effect of different blade design methods on the cavitation of a centrifugal pump and applying passive flow control with different impeller geometrical characteristics and operating conditions.

\section{References}

[1] R. Hirschi, P. Dupont, F. Avellan, J. N. Favre, J. F. Guelich, and E. Parkinson, "Centrifugal pump performance drop due to leading edge cavitation: numerical predictions compared with model tests," Journal of Fluids Engineering, vol. 120, no. 4, pp. 705-711, 1998.

[2] M. Hofmann, B. Stoffel, J. Friedrichs, and G. Kosyna, "Similarities and geometrical effects on rotating cavitation in two scaled centrifugal pumps," in Proceedings of the 4rth International Symposium on Cavitation (CAV '01), pp. 20-23, California Institute of Technology, Pasadena, Calif, USA, June 2001. 
[3] M. Frobenius, R. Schilling, J. Friedrichs, and G. Kosyna, "Numerical and experimental investigations of the cavitating flow in a centrifugal pump impeller," in Proceedings of the ASME Joint U.S.-European Fluids Engineering Conference (FEDSM '02), pp. 361-368, Montreal, Canada, July 2002.

[4] O. Coutier-Delgosha, R. Fortes-Patella, J. L. Reboud, M. Hofmann, and B. Stoffel, "Experimental and numerical studies in a centrifugal pump with two-dimensional curved blades in cavitating condition," Journal of Fluids Engineering, vol. 125, no. 6, pp. 970-978, 2003.

[5] V. Siljegovic, N. Milicevic, and P. Griss, "Passive, programmable flow control in capillary force driven microfluidic networks," in Proceedings of the 13th International Conference on Solid-State Sensors and Actuators and Microsystems (TRANSDUCERS '05), pp. 1565-1568, Seoul, Korea, June 2005.

[6] A. Ulas, "Passive flow control in liquid-propellant rocket engines with cavitating venturi," Flow Measurement and Instrumentation, vol. 17, no. 2, pp. 93-97, 2006.

[7] X. Escaler, E. Egusquiza, M. Farhat, F. Avellan, and M. Coussirat, "Detection of cavitation in hydraulic turbines," Mechanical Systems and Signal Processing, vol. 20, no. 4, pp. 983-1007, 2006.

[8] D. Japikse, K. N. Oliphant, and D. O. Baun, "Stability Enhancement of Compressors and Turbopumps by Passive Flow Control," Thermal and Fluids Analysis Works (TFAWS), College Park, MD, USA, 7-11 August 2006.

[9] X. Luo, Y. Zhang, J. Peng, H. Xu, and W. Yu, "Impeller inlet geometry effect on performance improvement for centrifugal pumps," Journal of Mechanical Science and Technology, vol. 22, no. 10, pp. 1971-1976, 2008.

[10] D. Wu, L. Wang, Z. Hao, Z. Li, and Z. Bao, "Experimental study on hydrodynamic performance of a cavitating centrifugal pump during transient operation," Journal of Mechanical Science and Technology, vol. 24, no. 2, pp. 575-582, 2010.

[11] S. D. Kyparissis and D. P. Margaris, "Experimental study in a centrifugal pump with double-arc synthetic blade design method in cavitating and non-cavitating conditions," in Proceedings of the 4th International Conference on Experiments / Process / System Modeling / Simulation / Optimization, (ICEpsMsO '11), Athens, Greece, July 2011.

[12] S. D. Kyparissis and D. P. Margaris, "Experimental investigation of cavitation in a centrifugal pump with doublearc synthetic blade design method," International Review of Mechanical Engineering, vol. 5, no. 5, pp. 884-892, 2011.

[13] S. D. Kyparissis and D. P. Margaris, "Experimental and computational study on the effects of the cavitation to the total head of a centrifugal pump," in Proceedings of the 5th International Conference from Scientific Computing to Computational Engineering (IC-SCCE '12), Athens, Greece, July 2012.

[14] S. D. Kyparissis, E. C. Douvi, E. E. Panagiotopoulos, D. P. Margaris, and A. E. Filios, "CFD flowfield analysis and hydrodynamic double-arc blade design effects for optimum centrifugal pump performance," International Review of Mechanical Engineering, vol. 3, no. 3, pp. 284-292, 2009.

[15] S. D. Kyparissis, E. C. Douvi, E. E. Panagiotopoulos, D. P. Margaris, and A. E. Filios, "Parametric study performance of a centrifugal pump based on simple and double-arc blade design methods," in Proceedings of the 3rd International Conference on Experiments / Process / System Modeling / Simulation / Optimization (IC-EpsMsO '09), pp. 8-11, Athens, Greece, July 2009.
[16] S. D. Kyparissis, D. P. Margaris, E. E. Panagiotopoulos, and A. E. Filios, "CFD analysis on the effects of the blade design method to the centrifugal pump performance," International Review of Mechanical Engineering, vol. 3, no. 5, pp. 553-561, 2009.

[17] C. Pfleiderer, Die Kreiselpumpen Für Flüssigkeiten Und Gase, Springer, Berlin, Germany, 1961.

[18] P. Girdhar and O. Moniz, Practical Centrifugal Pumps: Design, Operation and Maintenance, Elsevier, Oxford, UK, 2005. 

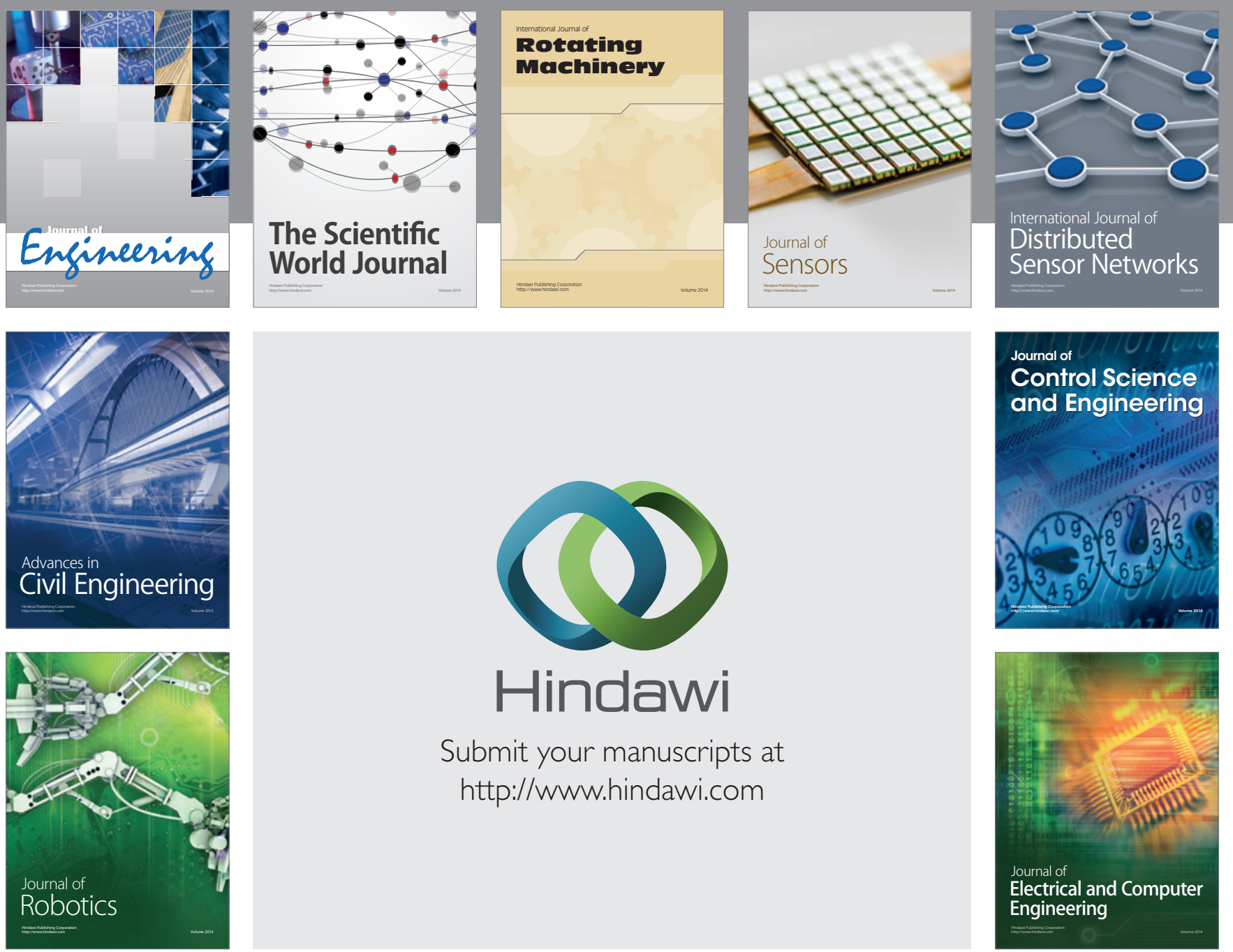

Submit your manuscripts at

http://www.hindawi.com
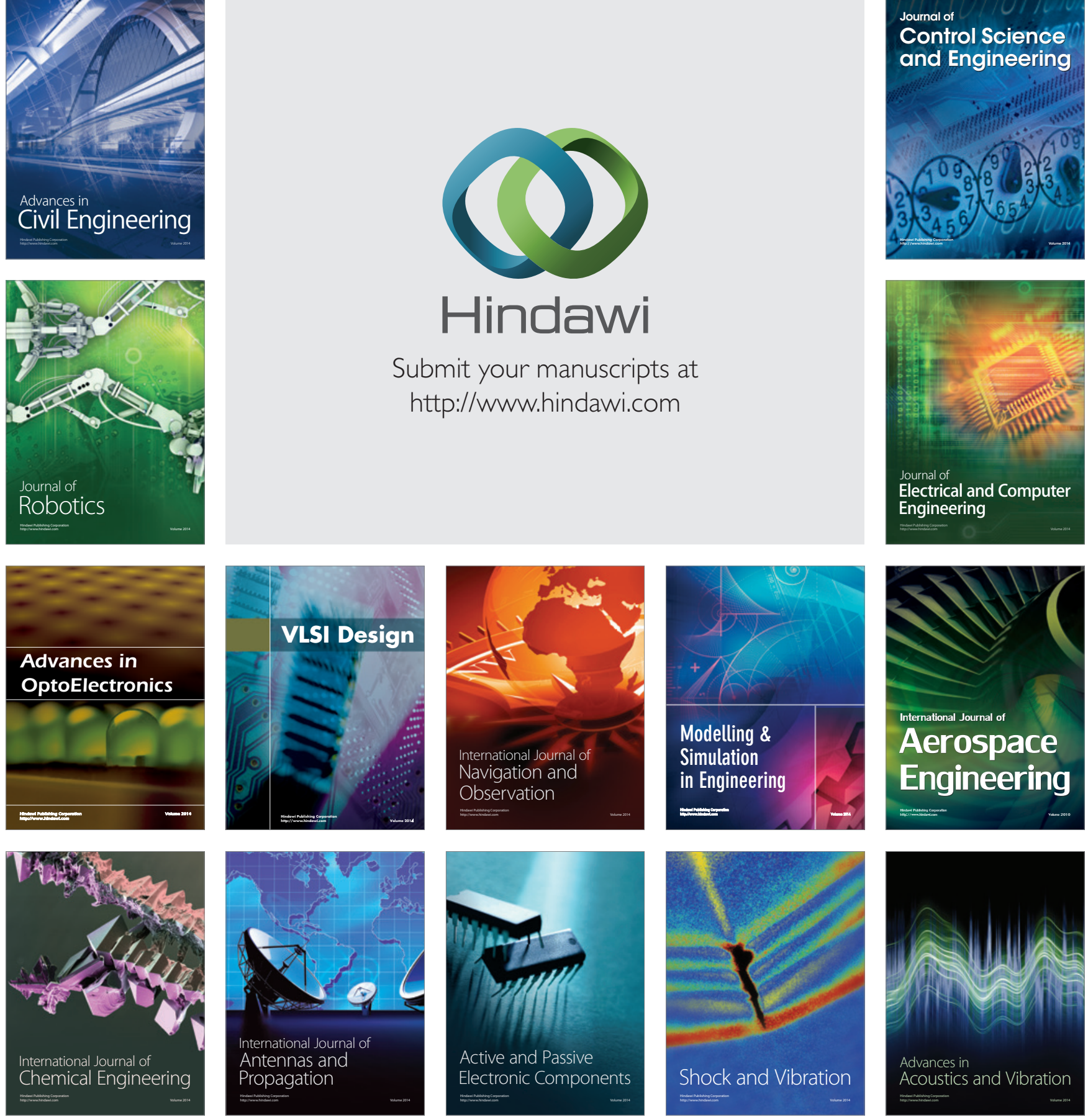